\title{
FURTHER PROPERTIES OF A RANDOM GRAPH WITH DUPLICATIONS AND DELETIONS
}

\author{
ÁGNES BACKHAUSZ AND TAMÁS F. MÓRI
}

\author{
mTA Alfréd Rényi Institute of Mathematics, Budapest and Department of \\ Probability Theory and Statistics, \\ EÖTVÖS LORÁND UNIVERSITY \\ Pázmány P. s. 1/C, H-1117 Budapest, Hungary \\ E-mail address: agnes@math.elte.hu \\ Department of Probability Theory and Statistics, \\ EÖTVÖS LORÁND UNIVERSITY \\ Pázmány P. s. 1/C, H-1117 Budapest, Hungary \\ E-mail address: mori@math.elte.hu
}

\begin{abstract}
We deal with a random graph model where at each step, a vertex is chosen uniformly at random, and it is either duplicated or its edges are deleted. Duplication has a given probability. We analyse the limit distribution of the degree of a fixed vertex, and derive a.s. asymptotic bounds for the maximal degree. The model shows a phase transition phenomenon with respect to the probabilities of duplication and deletion.
\end{abstract}

Keywords: scale free, duplication, deletion, random graphs, maximal degree.

\section{INTRODUCTION}

For researchers in mathematical biology it is evident that duplication of the information in the genome is a dominant evolutionary force in shaping biological networks. On the other hand, due to injuries, deletion of edges or vertices is also a phenomenon which is natural to consider. We analyse the random graph model that was described in a recent paper of Thörnblad [28, 2014+]. This model evolves in discrete time steps, and it has a parameter $0<\theta<1$. We start from a single vertex without edges. At each step, we choose a vertex $v$ uniformly at random. With probability $\theta$ we duplicate $v$; that is, we add a new vertex and connect it to the neighbours of $v$ and to $v$ itself with single edges. Otherwise, with probability $1-\theta$, all edges of $v$ are deleted (the vertex itself stays in the graph, and has the chance to get new edges later on).

As it was presented in [28] and as we will see later, the asymptotic behaviour of the model depends on the value of $\theta$, and there is a phase transition phenomenon. Naturally, our results will also be different for certain regimes of the duplication probability. The case $0<\theta<1 / 2$ is the subcritical case, where deletion is more likely, and as we will prove, the maximal degree has the order of logarithm of the actual number of vertices, almost surely. In the critical case $(\theta=1 / 2)$ the maximal degree grows faster; we have the square of the logarithm of the number of vertices. Finally, in the supercritical case, when $1 / 2<\theta<1$ and the duplication is dominant, the maximal degree will be compared to the number of vertices (without logarithm) in some sense. We remark that similar phase transition is present in some other

Date: 18 September 2014.

2010 Mathematics Subject Classification. 05C80, 60F15, 68 Q87.

Supported by the Hungarian Scientific Research Fund - OTKA K 108615. 
random graph models where deletion (but no duplication) is introduced; see e.g. the work of Vallier [30, 2013].

The random graph defined above consists of disjoint cliques. The vertices form separate clusters, and two vertices are connected if and only if they belong to the same cluster. Due to this simple structure, it may be interesting from the point of view of coagulation-fragmentation models or population dynamics. Champagnat, Lambert and Richard described certain properties of a continuous version of this model, the so-called splitting trees with mutations, where the clusters correspond to different alleles of a gene [6, 7, 2012], 8, 2013].

This shows that the current model does not have a fine structure as a graph. In the paper [1, 2014+] we described the connection of the critical case of the model analysed here to a model which is also built on duplication and deletion, but has a nontrivial graph structure. That model also turns out to be highly clustered: as one can expect, due to the duplication steps, some dense clusters evolve, while edges between clusters are rare. These highly clustered networks come up in mathematical biology, e.g. for modelling protein-protein interaction networks. Thus graph models with duplication (but with a richer graph structure than the actual one) may also be found in the literature. In most of those models edges are never deleted, but only some randomly chosen edges of the chosen vertex are duplicated, and some extra random edges may be added to the new vertex (Kim at al. [19, 2002], Pastor-Satorras, Smith and Solé [25. 2003], Chung et al. 9, 2003], Bebek et al. [4, 2006]). However, these papers did not contain mathematically rigorous arguments, and some results of the earlier ones (stating that the degree distribution is polynomially decaying with an exponential cutoff) were disclaimed by the latter ones. Recently, Hermann and Pfaffelhuber [17, 2014+] have proved several results on the frequency of isolated vertices and cliques, and also on the evolution of the degree of a fixed vertex in the initial graph. Various other models were also introduced, where the choice of the duplicated vertex is not uniform but depends on the degrees (Jordan [18, 2011], Cohen, Jordan and Voliotis [10, 2010], Farczadi and Wormald [13, 2014+]) or on the state of a hidden Markov chain (Hamdi, Krishnamurthy and Yin [16, 2013+]).

Notice that in this duplication-deletion model vertices of larger degree are more likely to increase their degree, because the probability that one of their neighbours is duplicated is larger. On the other hand, the probabilty of decreasing their degree is also larger. However, this is a kind of a preferential attachment phenomenon. Preferential attachment models are still popular for modelling web graphs or biological networks since the seminal paper of Albert and Barabási [3, 1999], and also from a theoretical point of view. It is worth mentioning the model free approaches of Ostroumova, Ryabchenko and Samosvat 24, 2013] and Dereich and Ortgiese [12, 2014]. However, for example, due to the possibility that the degree of a vertex can decrease to 1 in a single step by deletion, our model does not fit into those frameworks, in which the degree of a fixed vertex can not decrease. Maximal degree was also investigated in certain preferential attachment models, see e.g. [21, Móri, 2005]. Bubeck, Mossel and Rácz showed that the seed graph may have influence on the limiting distribution of the maximal degree in some kind of preferential attachment models. 5. 2014]. Since all vertices of the initial configuration are deleted after finitely many steps, this phenomenon does not occur in the current model.

Preferential attachment models are often investigated due to their scale free property: the proportion of vertices of degree $d$ tends to some contant $c_{d}$ in some sense, and $c_{d}$ decays polynomially as $d \rightarrow \infty$. The current model has a rather different asymptotic degree distribution, as the results of Thörnblad [28] and [1] 
shows. Since we will use it later on, we sum up these results, as follows. In accordance with [28] we introduce

$$
\beta=\frac{\theta}{2 \theta-1}, \quad \gamma=\frac{1-\theta}{\theta} .
$$

Theorem A. [1, B-M, 2014+, for $\theta=1 / 2$ ] 28, Thörnblad, 2014+, for $\theta \neq$ $1 / 2]$. Let $X[n, k]$ denote the proportion of vertices of degree $k$ after $n$ steps in the duplication-deletion model defined above. Then we have

$$
X[n, k] \rightarrow c_{k} \quad \text { almost surely as } n \rightarrow \infty,
$$

where $\left(c_{k}\right)_{k=1}^{\infty}$ is the unique sequence of positive numbers satisfying the following equations.

$$
c_{0}=\frac{1-\theta}{1+\theta}\left(1+c_{1}\right) ; \quad c_{k}=\frac{k+1}{k+1+\theta}\left(\theta c_{k-1}+(1-\theta) c_{k+1}\right) \quad(k \geq 1) .
$$

Furthermore, $\sum_{k=0}^{\infty} c_{k}=1$.

- Subcritical case. If $0<\theta<1 / 2$, then

$$
\begin{aligned}
& c_{k}=\gamma^{-k-1} \int_{0}^{1} \frac{t^{k+1}(1-t)^{-1-\beta}}{\left(1-\gamma^{-1} t\right)^{1-\beta}} d t \quad(k \geq 0), \text { and } \\
& c_{k} \sim(-\beta)^{-1}(1-\beta)^{-\beta} \Gamma(1-\beta) \gamma^{-k} k^{\beta} \quad \text { as } k \rightarrow \infty .
\end{aligned}
$$

- Critical case. For $\theta=1 / 2$ we have

$$
\begin{gathered}
c_{k}=(k+1) \int_{0}^{\infty} \frac{t^{k} e^{-t}}{(1+t)^{k+2}} d t \quad(k \geq 0), \text { and } \\
c_{k} \sim(e \pi)^{1 / 2} k^{1 / 4} e^{-2 \sqrt{k}} \quad \text { as } k \rightarrow \infty .
\end{gathered}
$$

- Supercritical case. If $1 / 2<\theta<1$, then

$$
\begin{gathered}
c_{k}=\gamma \int_{0}^{1} \frac{t^{k+1}(1-t)^{\beta-1}}{(1-\gamma t)^{1+\beta}} d t \quad(k \geq 0), \text { and } \\
c_{k} \sim \gamma \beta^{\beta} \Gamma(\beta+1) k^{-\beta} \quad \text { as } k \rightarrow \infty .
\end{gathered}
$$

(Note that our $c_{k}$ corresponds to Thörnblad's $d_{k+1}$.)

Therefore the asymptotic degree distribution decays exponentially in the subcritical case, polynomially in the supercritical case, and slower than exponential but faster than polynomial in the critical case.

The paper is built up as follows. In Section 2 we describe useful variants of the model and analyse the evolution of the number of vertices. Section 3 contains our results on the asymptotic behaviour of the degree of a fixed vertex. This is used in Section 4, where we give bounds for the maximal degree, which are valid with probability 1 . It will follow that the index of the vertex with maximal degree tends to infinity, that is, there is no persistent hub in this model.

\section{VARIANTS OF ThÖRNBLAD'S MODEL}

This is a discrete time model. Let us start from a single vertex. The graph is modified in two ways: at every step a vertex is selected at random, with equal probability, then this vertex is either duplicated or deleted. Duplication means that a new vertex is added to the graph, and it is connected to the selected vertex and its neighbours. Deletion means that the edges of the selected vertex get deleted, but not the vertex itself. Every step, independently of the past, is a duplication with probability $\theta$, and a deletion with probability $1-\theta(0<\theta<1)$. This model is called Version 1 or Thörnblad's model [28]. 
Further versions differ from Version 1 only by time transforms.

In Version 2 the development of the graph is slowed down. Let $N_{n-1}$ be the number of vertices of the graph after the $(n-1)$ th step. At the $n$th step, the graph is not modified with probability $1-N_{n-1} / n$. Otherwise each existing vertex has equal probability to be selected; the selected vertex (exactly one) is duplicated or deleted with probabilities $\theta$ and $1-\theta$, resp. All the above randomizations are independent of each other. As we will see, in this way the graph does not change in the majority of the steps.

Version 3 is defined in continuous time. Every vertex is given two clocks at its birth, which alarm according to independent homogeneous Poisson processes with rates $\theta$ and $1-\theta$. When the first clocks rings, the vertex gets duplicated, while the second clock determines deletion times. In this model, steps occur at an exponentially accelerating pace.

Focusing only on moments when something happens all versions look identical. This makes it possible to choose the most convenient version for different proofs.

In all versions, at every moment, the graph is a disjoint union of complete gaphs (cliques). This is obviously true in the beginning, and it is easy to see that neither duplication, nor deletion can break this property.

Lemma 1. Let us denote the number of vertices after $n$ steps by $N_{n}$, and in Version 3 , the size of the graph at time $t$ will be denoted by $N(t)$. Then a.s.

$$
\begin{aligned}
N_{n} & \sim \theta n & & \text { in Version } 1 ; \\
N_{n} & \sim \zeta n^{\theta} & & \text { in Version 2, where } \zeta \text { is a positive random variable; } \\
N(t) & \sim \eta e^{\theta t} & & \text { in Version } 3, \text { where } \eta \text { is a positive random variable. }
\end{aligned}
$$

Proof. The proof for Version 1 is obvious.

Version 2. Let $\mathcal{F}_{n}$ denote the $\sigma$-field generated by the first $n$ steps. At step $n$ the number of vertices increases by 1 with (conditional) probability $\theta N_{n-1} / n$. Thus

Introduce

$$
E\left(N_{n} \mid \mathcal{F}_{n-1}\right)=\left(1+\frac{\theta}{n}\right) N_{n-1}
$$

$$
\varkappa_{n}=\prod_{i=1}^{n}\left(1+\frac{\theta}{i}\right)^{-1}=\frac{\Gamma(1+\theta) \Gamma(n+1)}{\Gamma(n+1+\theta)} \sim \Gamma(1+\theta) n^{-\theta} .
$$

Then $\left(\varkappa_{n} N_{n}, \mathcal{F}_{n}\right)$ is a nonnegative martingale, which is known to be a.s. convergent. Let $\zeta=\lim _{n \rightarrow \infty} n^{-\theta} N_{n}$.

We still have to show that $\zeta>0$. Let $R_{n}=\left(N_{n}-1\right)^{-1}$, if $N_{n} \geq 2$, and $R_{n}=1$ otherwise. This time let

$$
\kappa_{n}=\prod_{i=1}^{n}\left(1-\frac{\theta}{i} I\left(N_{i-1} \geq 2\right)\right)^{-1} \asymp n^{\theta}
$$

as $n \rightarrow \infty$. Here $I(\cdot)$ stands for the indicator of the event in brackets. Clearly,

$$
E\left(R_{n} \mid \mathcal{F}_{n-1}\right)=\frac{1}{N_{n-1}} \cdot \frac{\theta N_{n-1}}{n}+\frac{1}{N_{n-1}-1}\left(1-\frac{\theta N_{n-1}}{n}\right)=\frac{1}{N_{n-1}-1}\left(1-\frac{\theta}{n}\right)
$$

on the event $\left\{N_{n-1} \geq 2\right\} \in \mathcal{F}_{n-1}$, and $=1$ on its complement. Hence $\left(\kappa_{n} R_{n}, \mathcal{F}_{n}\right)$ is a nonnegative martingale. Consequently, $n^{\theta} / N_{n}$ converges a.s., and its limit is obviously $1 / \zeta$.

Version 3. $N\left(\theta^{-1}\right)$ is a Yule process (see e.g. [26, 29]), thus it is geometrically distributed, namely $\operatorname{Geom}\left(e^{-\theta t}\right)$, and $N(t) \sim \eta e^{\theta t}$, where $\eta$ is an exponential random variable of expected value 1 . 


\section{The DEGREE PROCESS OF A FIXED VERTEX}

In this section we consider Version 3, because, as we will see, that is the most natural choice for the individual degree processes. Of course, the results of this section can easily be transferred to Version 1, by using Lemma 1.

Let the vertices be labelled by $1,2, \ldots$ in the order of birth, and let $d_{i}(t)$ denote the degree of vertex $i$ at time $t$.

Theorem 1. For every $i=1,2, \ldots$ we have

$$
\lim _{t \rightarrow \infty} P\left(d_{i}(t)=k\right)=q_{k}, \quad k=0,1, \ldots,
$$

where

$$
q_{0}=\gamma\left(1-c_{0}\right), \quad q_{k}=c_{k-1}-\gamma c_{k}, k=1,2, \ldots,
$$

where the sequence $\left(c_{k}\right)$ is defined in Theorem A. Here $q_{k}>0, k=0,1, \ldots$, and $\sum_{k=0}^{\infty} q_{k}=1$.

Proof. Let us fix a vertex. Clearly, its degree is a continuous time Markov process with infinitesimal generator

$$
\mathbf{M}=\left[\begin{array}{ccc}
1-\theta & 0 & \ldots \\
1-\theta & 0 & \ldots \\
\vdots & \vdots &
\end{array}\right]+\left[\begin{array}{cccccc}
-1 & \theta & & & & \\
1-\theta & -2 & 2 \theta & & & \\
& 2(1-\theta) & -3 & 3 \theta & & \\
& & 3(1-\theta) & -4 & 4 \theta & \\
& & & \ddots & \ddots & \ddots
\end{array}\right]
$$

The process is positive recurrent, because deletion cuts back the degree to 0 at a constant rate. Hence it has a stationary distribution $q=\left(q_{0}, q_{1}, \ldots\right)$ which is the unique discrete distribution satisfying $q \mathbf{M}=0$ [26]. Thus,

$$
q_{0}=(1-\theta)\left(1+q_{1}\right), \quad q_{k}=\frac{k \theta q_{k-1}+(k+1)(1-\theta) q_{k+1}}{k+1}, k \geq 1 .
$$

From Theorem A it follows that the numbers $q_{k}$ in (11) satisfy (2). They sum up to 1 , because

$$
\sum_{k=0}^{\infty} q_{k}=\gamma\left(1-c_{0}\right)+\sum_{k=1}^{\infty}\left(c_{k-1}-\gamma c_{k}\right)=\gamma+(1-\gamma) \sum_{k=0}^{\infty} c_{k}=1
$$

Finally, their positivity follows from the integral form of $c_{k}$, which can be found in 1] for the critical case, and in 28] for the subcritical and supercritical cases; see Theorem A. Namely, we immediately obtain in the subcritical case

$$
q_{k}=\gamma^{-k} \int_{0}^{1} \frac{t^{k}(1-t)^{-\beta}}{\left(1-\gamma^{-1} t\right)^{1-\beta}} d t
$$

and in the supercritical case

$$
q_{k}=\gamma \int_{0}^{1} \frac{t^{k}(1-t)^{\beta-1}}{(1-\gamma t)^{\beta}} d t
$$

for $k \geq 1$. In the critical case, by equation (2) and partial integration we get

$$
\begin{aligned}
q_{k} & =k \int_{0}^{\infty} \frac{t^{k-1} e^{-t}}{(1+t)^{k+1}} d t-(k+1) \int_{0}^{\infty} \frac{t^{k} e^{-t}}{(1+t)^{k+2}} d t \\
& =k \int_{0}^{\infty} \frac{t^{k-1} e^{-t}}{(1+t)^{k+1}} d t+\left[\frac{t^{k} e^{-t}}{(1+t)^{k+1}}\right]_{0}^{\infty}-\int_{0}^{\infty} \frac{k t^{k-1} e^{-t}-t^{k} e^{-t}}{(1+t)^{k+1}} d t \\
& =\int_{0}^{\infty} \frac{t^{k} e^{-t}}{(1+t)^{k+1}} d t .
\end{aligned}
$$


It is somewhat surprising. In spite that the limit distribution of the degree is the same for every vertex, the asymptotic degree distribution of the graph is different. If the the degrees were independent and identically distributed, the proportion of vertices with fixed degree would converge to the corresponding probability. In our model neither condition is satisfied, not even approximately. On one hand, in Version 3, the size of the graph grows exponentially. Consequently, at every moment the vast majority of the vertices are relatively young, so the limit distribution cannot be applied to them. On the other hand, if the vertices were nearly independent, the number of vertices with high degree would follow the Poisson distribution; but in our model, if there exists at least one vertex of a large degree $d$, then all its neighbours have the same degree, therefore many of such vertices are coexistent.

This phenomenon can be better understood if we consider the degree process of an arbitrary vertex. The higher the degree is, the shorter it sustains. Therefore a reversed size biased sampling can be observed: at a given moment the probability that a given vertex has degree $d$ is less than the proportion of degree $d$ ones among all vertices.

In Section 4 we shall need asymptotics for the tail of the stationary distribution.

\section{Theorem 2.}

(3) Subcritical case. $\quad q_{k}+q_{k+1}+\cdots \sim(1-\beta)^{2-\beta} \Gamma(1-\beta) k^{\beta-1} \gamma^{-k}$;

(4) Critical case. $\quad q_{k}+q_{k+1}+\cdots \sim(e \pi)^{1 / 2} k^{1 / 4} e^{-2 \sqrt{k}}$;

(5) Supercritical case. $q_{k}+q_{k+1}+\cdots \sim \gamma \beta^{\beta} \Gamma(\beta-1) k^{1-\beta}$,

as $k \rightarrow \infty$.

Proof. In the subcritical case we have

$$
\sum_{j=k}^{\infty} q_{j}=\gamma^{-k} \int_{0}^{1} \frac{t^{k}(1-t)^{-\beta}}{\left(1-\gamma^{-1} t\right)^{2-\beta}} d t
$$

while in the supercritical case

$$
\sum_{j=k}^{\infty} q_{j}=\gamma \int_{0}^{1} \frac{t^{k}(1-t)^{-2+\beta}}{(1-\gamma t)^{\beta}} d t .
$$

If $k \rightarrow \infty$, both integrals become relatively negligible over any interval $[0,1-\varepsilon]$, compared to those over $[1-\varepsilon, 1]$. Hence in the denominators we can replace $t$ with 1 , thus reducing to complete beta integrals.

In the critical case $q_{k}+q_{k+1}+\cdots=c_{k-1}$, hence Theorem A can be applied.

Obviously, every vertex becomes isolated infinitely many times, due to deletion. What can be said about the extremely high degrees?

\section{Theorem 3.}

$$
\begin{array}{ll}
\text { Subcritical case. } & \limsup _{t \rightarrow \infty} \frac{d_{i}(t)}{\log \log N(t)}=\frac{1}{\log \gamma} ; \\
\text { Critical case. } & \limsup _{t \rightarrow \infty} \frac{d_{i}(t)}{(\log \log N(t))^{2}}=1, \\
\text { Supercritical case. } & \limsup _{t \rightarrow \infty} \frac{\log d_{i}(t)}{\log \log N(t)}=\frac{1}{\beta-1} .
\end{array}
$$

for $i=1,2, \ldots$.

Proof. First we investigate how large can the degree grow between two consecutive deletions. Let $p_{i}(r)$ denote the probability that a vertex of degree $i$ will 
sometimes have degree $r$ at least once before it is selected for deletion. Then we clearly have

$$
\begin{aligned}
& p_{0}(r)=\theta p_{1}(r) ; \\
& p_{i}(r)=\frac{(i+1) \theta p_{i+1}(r)+i(1-\theta) p_{i-1}(r)}{i+1}, \quad i=1,2, \ldots, r-1 ; \\
& p_{r}(r)=1 .
\end{aligned}
$$

Introduce $a_{i}=p_{i}(r) / p_{0}(r)$; it does not depend on $r$ provided $r>i$. The probability we are interested in is $p_{0}(r)=1 / a_{r}$. The sequence $\left(a_{r}\right)_{r \geq 0}$ satisfies the following recursion.

$$
a_{0}=1, \quad a_{1}=\frac{1}{\theta}, \quad a_{i}=\theta a_{i+1}+\frac{i}{i+1}(1-\theta) a_{i-1}, \quad i=1,2, \ldots .
$$

In the critical case we can use some well-known facts about Laguerre polynomials $L_{r}(x)$ [27. They can be defined by the following recursion formula.

(7) $L_{0}(x)=1, L_{1}(x)=1-x$,

$$
L_{r+1}(x)=\frac{(2 r+1-x) L_{r}(x)-r L_{r-1}(x)}{r+1}, r=1,2, \ldots .
$$

Their asymptotic behaviour for large $r$ and fixed $y>0$ is given by

$$
L_{r}(-y) \sim 2^{-1} \pi^{-1 / 2} r^{-1 / 4} e^{-y / 2} y^{-1 / 4} e^{2 \sqrt{y r}} .
$$

Recursions (6) and (7) coincide if $\theta=1 / 2$ and $x=-1$. Hence we obtain

$$
p_{0}(r)=\frac{1}{a_{r}}=\frac{1}{L_{r}(-1)} \sim 2 \sqrt{e \pi} r^{1 / 4} e^{-2 \sqrt{r}}
$$

in the critical case.

If $\theta \neq 1 / 2$, we can analyse the asymptotic behaviour of the sequence $\left(a_{r}\right)$ by computing its generating function $G(z)=\sum_{r=0}^{\infty} a_{r} z^{r}$. From (6) it follows that

$$
\sum_{r=1}^{\infty}(r+1) a_{r} z^{r}=(1-\theta) \sum_{r=1}^{\infty} r a_{r-1} z^{r}+\theta \sum_{r=1}^{\infty}(r+1) a_{r+1} z^{r}
$$

that is,

$$
(z G(z))^{\prime}-1=(1-\theta) z(z G(z))^{\prime}+\theta\left(G^{\prime}(z)-\frac{1}{\theta}\right) .
$$

This leads to the following homogeneous linear ODE.

$$
\left(\theta-z+(1-\theta) z^{2}\right) G^{\prime}(z)=(1-(1-\theta) z) G(z), \quad G(0)=1 .
$$

Its solution can easily be expanded into a power series.

$$
G(z)=(1-z)^{-\beta}(1-\gamma z)^{\beta-1}=\sum_{r=0}^{\infty}(-z)^{r} \sum_{i=0}^{r}\left(\begin{array}{c}
\beta-1 \\
i
\end{array}\right)\left(\begin{array}{c}
-\beta \\
r-i
\end{array}\right) \gamma^{i}
$$

Thus,

$$
a_{r}=(-1)^{r} \sum_{i=0}^{r}\left(\begin{array}{c}
\beta-1 \\
i
\end{array}\right)\left(\begin{array}{c}
-\beta \\
r-i
\end{array}\right) \gamma^{i}
$$

Suppose first that $\theta>1 / 2$. Then $\gamma<1$ and $\beta>1$. Since

$$
(-1)^{r} r^{1-\beta}\left(\begin{array}{c}
-\beta \\
r
\end{array}\right)=\frac{r^{1-\beta} \Gamma(r+\beta)}{\Gamma(r+1) \Gamma(\beta)}
$$

converges as $r \rightarrow \infty$, therefore it is bounded. Consequently, we have

$$
\left|(-1)^{r} r^{1-\beta}\left(\begin{array}{c}
\beta-1 \\
i
\end{array}\right)\left(\begin{array}{c}
-\beta \\
r-i
\end{array}\right) \gamma^{i}\right| \leq b_{i}
$$


uniformly in $r \geq i$, where the infinite series $\sum b_{i}$ converges. Hence

$$
\begin{aligned}
\lim _{r \rightarrow \infty} r^{1-\beta} a_{r}=\sum_{i=0}^{\infty} \lim _{r \rightarrow \infty}(-1)^{r} r^{1-\beta}\left(\begin{array}{c}
\beta-1 \\
i
\end{array}\right)\left(\begin{array}{c}
-\beta \\
r-i
\end{array}\right) \gamma^{i} & \\
= & \frac{1}{\Gamma(\beta)} \sum_{i=0}^{\infty}\left(\begin{array}{c}
\beta-1 \\
i
\end{array}\right)(-\gamma)^{i}=\frac{(1-\gamma)^{\beta-1}}{\Gamma(\beta)}=\frac{\beta^{1-\beta}}{\Gamma(\beta)},
\end{aligned}
$$

and, by this,

$$
p_{0}(r) \sim \beta^{\beta-1} \Gamma(\beta) r^{1-\beta}
$$

in the supercritical case.

The subcritical case is easy to reduce to the supercritical one. Let $a_{r}^{\prime}=\gamma^{-r} a_{r}$. Then $a_{r}^{\prime}$ satisfies the same recursion that $a_{r}$ does when $\theta$ is replaced by $1-\theta$. This substitution transforms the subcritical case into the supercritical one, furthermore, $\beta$ changes to $1-\beta$. Hence we get

$$
p_{0}(r)=\gamma^{-r}\left(a_{r}^{\prime}\right)^{-1} \sim(1-\beta)^{-\beta} \Gamma(1-\beta) r^{\beta} \gamma^{-r} .
$$

Up to time $t$ there are $(1-\theta) t(1+o(1)) \sim \gamma \log N(t)$ epochs (time intervals between consecutive deletions), hence $\max \left\{d_{i}(s): s \leq t\right\}$ is asymptotically equal to the maximum of $(1+o(1)) \gamma \log N(t)$ i.i.d. random variables with distribution $P(\xi \geq$ $r)=p_{0}(r)$. Starting from (11), (9) and (10), standard Borel-Cantelli arguments yield

in the subcritical case,

$$
\max \left\{d_{i}(s): s \leq t\right\} \sim \frac{\log \log N(t)}{\log \gamma}
$$

$$
\max \left\{d_{i}(s): s \leq t\right\} \sim \log ^{2} t \sim(\log \log N(t))^{2}
$$

in the critical case, and

$$
\log \max \left\{d_{i}(s): s \leq t\right\} \sim \frac{\log \log N(t)}{\beta-1}
$$

in the supercritical case, completing the proof. (Alternatively, one can apply [15, Theorem 4.4.4].)

\section{Maximal Degree}

Let $M_{n}$ denote the maximal degree in Version 1 after $n$ steps. From Theorem A it is clear that $M_{n} \rightarrow \infty$. In many scale-free random graph processes the order of magnitude of the maximal degree $M_{n}$ can be characterized in the following way: $M_{n} \asymp \min \left\{d: N_{n} c_{d}<1\right\}$, where $\left(c_{d}\right)$ is the asymptotic degree distribution, and $N_{n}$ is the size of the graph, see e.g. [21, 2005], [22, 2007], [23, 2010], [2, 2014]. This would give $M_{n} \asymp \log N_{n}$ in the subcritical case, $M_{n} \asymp \log ^{2} N_{n}$ in the critical one, and $M_{n} \asymp N_{n}^{1 / \beta}$ in the supercritical one. We will show that this estimate is valid in the subcritical and critical cases, but in the supercritical case we can prove less.

\section{Theorem 4.}

$$
\begin{array}{ll}
\text { Subcritical case. } & \frac{1-\theta}{\log \gamma} \leq \liminf _{n \rightarrow \infty} \frac{M_{n}}{\log N_{n}} \leq \limsup _{n \rightarrow \infty} \frac{M_{n}}{\log N_{n}} \leq \frac{1+\theta}{\theta \log \gamma} . \\
\text { Critical case. } & \frac{1}{16} \leq \liminf _{n \rightarrow \infty} \frac{M_{n}}{\log ^{2} N_{n}} \leq \limsup _{n \rightarrow \infty} \frac{M_{n}}{\log ^{2} N_{n}} \leq \frac{9}{4} . \\
\text { Supercritical case. } & \frac{\theta}{\beta} \leq \liminf _{n \rightarrow \infty} \frac{\log M_{n}}{\log N_{n}} \leq \limsup _{n \rightarrow \infty} \frac{\log M_{n}}{\log N_{n}} \leq \frac{1}{\beta} .
\end{array}
$$




\section{Proof of the upper bounds.}

The proof will be given for Version 2.

Let $d_{i}(n)$ denote the degree of vertex $i$ after step $n, i=1, \ldots, N_{n}$, where $N_{n}$ is the size of the graph after $n$ steps. Introduce

$$
S_{n}(r)=\sum_{i=1}^{N_{n}}\left(\begin{array}{c}
d_{i}(n) \\
r
\end{array}\right)
$$

Lemma 2. For every $n=1,2, \ldots$ and $r=0,1,2, \ldots$ we have

(12) Subcritical case.

$$
E S_{n}(r) \leq 2(r+1)(-\beta)^{r} n^{\theta}
$$

(13) Critical case.

$$
E S_{n}(r) \leq 2(r+1) ! \sqrt{n}
$$

$$
\text { Supercritical case. } E S_{n}(r) \leq \begin{cases}C_{r}(\theta) n^{\theta}, & \text { if } r<\beta-1 \\ C_{r}(\theta) n^{\theta}(1+\log n), & \text { if } r=\beta-1 \\ C_{r}(\theta) n^{(r+1)(2 \theta-1)}, & \text { if } r>\beta-1 .\end{cases}
$$

where $C_{r}(\theta)$ is a constant depending on $r$ and $\theta$ but independent of $n$..

Proof. First we will verify the following recursion. For every $n=1,2, \ldots$ and $r=1,2, \ldots$ we have

$$
E S_{n}(r)=\left(1+\frac{(2 \theta-1)(r+1)}{n}\right) E S_{n-1}(r)+\frac{\theta(r+1)}{n} E S_{n-1}(r-1) .
$$

At the $n$th step the $i$ th term of $S_{n}(r)$ can change in the following way. With the notation $d=d_{i}(n-1)$,

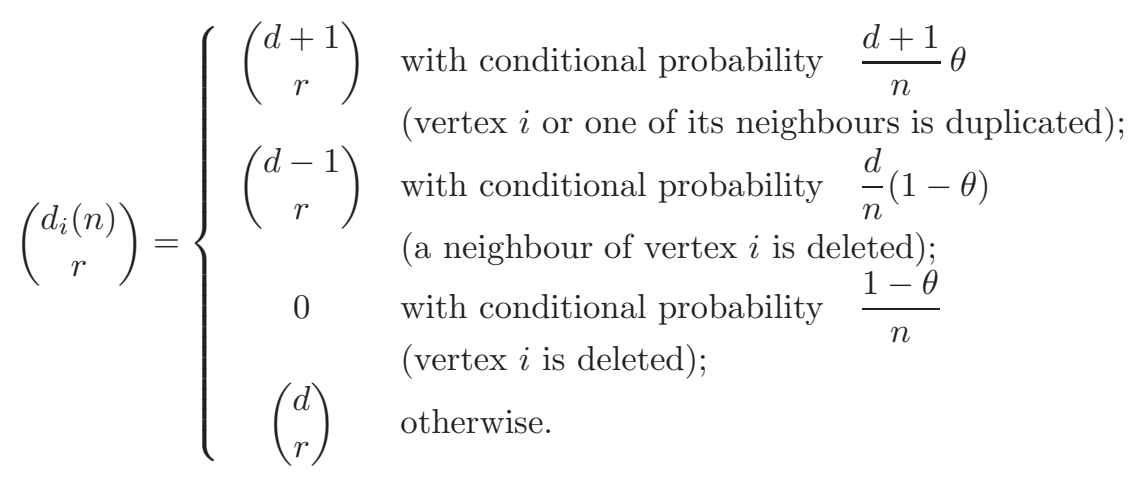

Thus,

$$
E\left(\left(\begin{array}{c}
d_{i}(n) \\
r
\end{array}\right) \mid \mathcal{F}_{n-1}\right)=\left(\begin{array}{c}
d \\
r
\end{array}\right)\left(1-\frac{d+1}{n}\right)+\left(\begin{array}{c}
d+1 \\
r
\end{array}\right) \frac{d+1}{n} \theta+\left(\begin{array}{c}
d-1 \\
r
\end{array}\right) \frac{d}{n}(1-\theta) .
$$

Besides, when vertex $i$ is duplicated, an additional term $\left(\begin{array}{c}d+1 \\ r\end{array}\right)$ also appears as the yield of the new vertex. Hence the total contribution of vertex $i$ in $E\left(S_{n}(r) \mid \mathcal{F}_{n-1}\right)$ is

$$
\begin{aligned}
\left(\begin{array}{l}
d \\
r
\end{array}\right)\left(1-\frac{d+1}{n}\right)+\left(\begin{array}{c}
d+1 \\
r
\end{array}\right) & \frac{d+2}{n} \theta+\left(\begin{array}{c}
d-1 \\
r
\end{array}\right) \frac{d}{n}(1-\theta) \\
= & \left(\begin{array}{l}
d \\
r
\end{array}\right)\left(1+\frac{(2 \theta-1)(r+1)}{n}\right)+\left(\begin{array}{c}
d \\
r-1
\end{array}\right) \frac{\theta(r+1)}{n} .
\end{aligned}
$$

This implies that

$$
E\left(S_{n}(r) \mid \mathcal{F}_{n-1}\right)=\left(1+\frac{(2 \theta-1)(r+1)}{n}\right) S_{n-1}(r)+\frac{\theta(r+1)}{n} S_{n-1}(r-1),
$$


as needed.

Next, we prove the lemma by double induction over $r$ and $n$, basing on the recursion (15).

Clearly, $S_{n}(0)=N_{n}$. From the proof of Lemma 1 we know that

$$
E N_{n}=\prod_{i=1}^{n}\left(1+\frac{\theta}{i}\right) \leq 2 \prod_{i=1}^{n-1}\left(1+\frac{\theta}{i}\right) \leq 2 \prod_{i=1}^{n-1}\left(1+\frac{1}{i}\right)^{\theta}=2 n^{\theta}
$$

in all three cases. Furthermore, $E S_{1}(1)=2 \theta$ and $E S_{1}(r)=0$ if $r>1$. Thus, for all pairs $(n, 0)$ and $(1, r)$ Lemma 2 holds true.

Let us check the induction step.

In the subcritical case, by using the induction hypothesis we can write

$$
\begin{aligned}
E S_{n}(r) \leq 2(r+1)(-\beta)^{r}(n & -1)^{\theta}\left(1+\frac{(r+1) \theta}{n \beta}\right) \\
& +2 r(-\beta)^{r-1}(n-1)^{\theta} \frac{\theta(r+1)}{n} \leq 2(r+1)(-\beta)^{r} n^{\theta},
\end{aligned}
$$

as needed.

In the critical case we have

$$
\begin{aligned}
& E S_{n}(r)=E S_{n-1}(r)+\frac{r+1}{2 n} E S_{n-1}(r-1) \\
& \quad \leq 2(r+1) ! \sqrt{n-1}\left(1+\frac{1}{2 n}\right) \leq 2(r+1) ! \sqrt{n} .
\end{aligned}
$$

Finally, in the supercritical case $C_{0}(\theta)=2$ will do. Suppose we have proved inequality (14) for $r-1$ (and all $n$ ). Introduce $s=(r+1)(2 \theta-1)$ and

$$
\kappa_{n}=\frac{\Gamma(n+1+s)}{\Gamma(n+1)},
$$

then

$$
\frac{\kappa_{n}}{\kappa_{n-1}}=1+\frac{s}{n} \leq\left(\frac{n}{n-1}\right)^{s},
$$

because $1+\frac{s}{n}<\left(1+\frac{1}{n}\right)^{s} \leq\left(1+\frac{1}{n-1}\right)^{s}=\left(\frac{n}{n-1}\right)^{s}$, if $s \geq 1$, and $1+\frac{s}{n} \leq\left(1-\frac{s}{n}\right)^{-1} \leq$ $\left(1-\frac{1}{n}\right)^{-s}=\left(\frac{n}{n-1}\right)^{s}$, if $0<s<1$. Hence, for $1 \leq j \leq n$ we have

$$
\frac{\kappa_{n}}{\kappa_{j}} \leq\left(\frac{n}{j}\right)^{s}
$$

By iterating equation (15) we get

$$
\begin{aligned}
\frac{E S_{n}(r)}{\kappa_{n}}=\frac{E S_{n-1}(r)}{\kappa_{n-1}}+\frac{(r+1) \theta}{n \kappa_{n}} E S_{n-1}(r-1) & =\ldots \\
& =\frac{E S_{1}(r)}{\kappa_{1}}+(r+1) \theta \sum_{j=2}^{n} \frac{E S_{j-1}(r-1)}{j \kappa_{j}},
\end{aligned}
$$

hence, by (16),

$$
E S_{n}(r) \leq n^{s} E S_{1}(r)+(r+1) \theta n^{s} \sum_{j=1}^{n-1} j^{-s} E S_{j}(r-1)=A+B
$$

In the right-hand side $A$ vanishes if $r>1$. For $r=1$ it is equal to $2 \theta n^{2(2 \theta-1)}$, which satisfies (14) in all three cases. Let us turn to $B$. 
First, suppose $r<\beta-1$, that is, $\theta>s$. Then, by the induction hypothesis we have

$$
B \leq(r+1) \theta C_{r-1}(\theta) n^{s} \sum_{j=1}^{n-1} j^{\theta-s-1} \leq \frac{(r+1) \theta}{\theta-s} C_{r-1}(\theta) n^{\theta} .
$$

Next, let $r=\beta-1$, that is, $\theta=s$. Then again

$$
B \leq(r+1) \theta C_{r-1}(\theta) n^{\theta} \sum_{j=1}^{n-1} \frac{1}{j} \leq(r+1) \theta C_{r-1}(\theta) n^{\theta}(1+\log n) .
$$

If $\beta-1<r \leq \beta$, that is, $r(2 \theta-1) \leq \theta<s$, then

$$
B \leq(r+1) \theta C_{r-1}(\theta) n^{s} \sum_{j=1}^{n-1} j^{\theta-s-1}(1+\log j) \leq(r+1) \theta C_{r-1}(\theta) Q n^{s},
$$

where

$$
Q=\sum_{j=1}^{\infty} j^{\theta-s-1}(1+\log j)<\infty
$$

Finally, if $\beta<r$, then

$$
B \leq(r+1) \theta C_{r-1}(\theta) n^{s} \sum_{j=1}^{n-1} j^{-2 \theta} \leq(r+1) \theta C_{r-1}(\theta) \zeta(2 \theta) n^{s},
$$

where $\zeta($. ) is the Riemann zeta function.

Let us continue the proof of Theorem 4

In the subcritical case, let us fix $z$ and $a$ in such a way that $0<z<-1 / \beta$, and $a \log (1+z)>1+\theta$. Then, by Lemma 2 we have

$$
\begin{array}{r}
E\left(\sum_{i=1}^{N_{n}}(1+z)^{d_{i}(n)}\right)=E\left(\sum_{i=1}^{N_{n}} \sum_{r=0}^{n}\left(\begin{array}{c}
d_{i}(n) \\
r
\end{array}\right) z^{r}\right)=E\left(\sum_{r=0}^{n} \sum_{i=1}^{N_{n}}\left(\begin{array}{c}
d_{i}(n) \\
r
\end{array}\right) z^{r}\right) \\
=\sum_{r=0}^{n} E S_{n}(r) z^{r} \leq 2 \sum_{r=0}^{\infty}(r+1)(-\beta z)^{r} n^{\theta}=\frac{2 n^{\theta}}{(1+\beta z)^{2}}=K n^{\theta} .
\end{array}
$$

By the Markov inequality,

$$
\begin{aligned}
& P\left(M_{n} \geq a \log n\right)=P\left((1+z)^{M_{n}} \geq(1+z)^{a \log n}\right) \\
& \leq n^{-a \log (1+z)} E\left(\sum_{i=1}^{N_{n}}(1+z)^{d_{i}(n)}\right) \leq K n^{\theta-a \log (1+z)} .
\end{aligned}
$$

The infinite sum of the right-hand side is convergent as $n$ runs through the positive integers, thus the Borel-Cantelli lemma implies $M_{n}<a \log n$ a.s. for every sufficiently large $n$. Consequently,

$$
\limsup _{n \rightarrow \infty} \frac{M_{n}}{\log n} \leq \frac{1+\theta}{\log \left(1-\beta^{-1}\right)}=\frac{1+\theta}{\log \gamma} .
$$

From Lemma 1 we know that $\log N_{n} \sim \theta \log n$ as $n \rightarrow \infty$.

In the critical case we can make use of Laguerre polynomials again. Their explicit form is

$$
L_{k}(y)=\sum_{r=0}^{k}\left(\begin{array}{l}
k \\
r
\end{array}\right) \frac{(-y)^{r}}{r !}
$$


Since the multiplicity of the maximal degree is at least $M_{n}+1$, we have

$$
S_{n}(r-1) \geq\left(M_{n}+1\right)\left(\begin{array}{c}
M_{n} \\
r-1
\end{array}\right)=r\left(\begin{array}{c}
M_{n}+1 \\
r
\end{array}\right) .
$$

Therefore, by Lemma 2

$$
E\left(\begin{array}{c}
M_{n} \\
r
\end{array}\right) \leq \frac{1}{r} E S_{n}(r-1) \leq 2 r ! \sqrt{n},
$$

for $r=1,2, \ldots$, hence

$$
E\left(L_{M_{n}}(-y)\right)=E\left(\sum_{r=0}^{n}\left(\begin{array}{c}
M_{n} \\
r
\end{array}\right) \frac{y^{r}}{r !}\right) \leq 1+2 \sqrt{n} \sum_{r=1}^{n} y^{r} \leq \frac{2 \sqrt{n}}{1-y}
$$

if $0<y<1$. Let $k=k_{n} \geq a \log ^{2} n$, where $y a>9 / 16$. Then the Markov inequality, combined with (8), implies

$$
\begin{aligned}
P\left(M_{n} \geq k\right)=P\left(L_{M_{n}}(-y) \geq L_{k}(-y)\right) & \leq \frac{E\left(L_{M_{n}}(-y)\right)}{L_{k}(-y)} \\
= & O\left(\sqrt{n} e^{-2(1+o(1)) \sqrt{y k}}\right)=O\left(n^{1 / 2-2 \sqrt{y a}}\right) .
\end{aligned}
$$

The exponent in $O($.$) is less than -1$, hence it makes a convergent series again, and from the Borel-Cantelli lemma

$$
\limsup _{n \rightarrow \infty} \frac{M_{n}}{\log ^{2} n} \leq a
$$

follows for every $a>9 / 16$. This time $\log n \sim 2 \log N_{n}$ by Lemma 1.

Finally, let us turn to the supercritical case. Let $a>2 \theta-1$ and $r$ so large that $r>\beta-1$ and $r(2 \theta-1-a)<-1$ hold. Then by the Markov inequality and (14) we have

$$
\begin{aligned}
P\left(M_{n} \geq n^{a}\right)=P\left(\left(\begin{array}{c}
M_{n} \\
r
\end{array}\right) \geq\left(\begin{array}{c}
n^{a} \\
r
\end{array}\right)\right) \leq \frac{E\left(\begin{array}{c}
M_{n} \\
r
\end{array}\right)}{\left(\begin{array}{c}
n^{a} \\
r
\end{array}\right)} & \\
\leq & \frac{E S_{n}(r-1)}{r\left(\begin{array}{c}
n^{a} \\
r
\end{array}\right)}=O\left(n^{r(2 \theta-1-a)}\right) .
\end{aligned}
$$

The proof can be completed with the help of the Borel-Cantelli lemma and Lemma 1 .

\section{Proof of the lower bounds.}

The proof will be performed for Version 3 . Let $\varepsilon$ be an arbitrarily small fixed positive number. The proof will consist of the following steps.

We first show that at time $(1-\theta) \sqrt{n}$ there are quite many isolated points in the graph. Clearly, they behave independently of each other after time $(1-\theta) \sqrt{n}$.

Then we give a lower bound for the probability that such an isolated vertex increases its degree above $k_{n}$ by time $\sqrt{n}$, where $k_{n}$ is an increasing positive sequence depending on $\theta$. It will follow that the probability that none of them can do it is so small that its sum over $n$ is convergent. Hence the Borel-Cantelli lemma implies that a.s. $M(\sqrt{n}) \geq k_{n}$ if $n$ is large enough.

Finally, we will show that the probability that a vertex having such a high degree at time $t_{n}$ will lose from its degree at least $\varepsilon k_{n}$ times in the interval $[\sqrt{n}, \sqrt{n+1}]$ is very small: it is also finitely summable. Thus, with $n=\left\lfloor t^{2}\right\rfloor$ we have a.s. $M(t) \geq(1-\varepsilon) k_{n}$ if $t$ is large enough.

In more details, let us start with the number of isolated vertices. For the sake of brevity denote $N((1-\theta) \sqrt{n}-1)$ by $N_{n}$. For each vertex present at time $(1-\theta) \sqrt{n}-1$, 
the probability that, during the next time unit, it will be deleted some time and not duplicated after that is

$$
\sigma=(1-\theta)\left(1-e^{-1}\right)
$$

(Something happens to the vertex, and the last event is a deletion.) Therefore the number of isolated vertices at time $(1-\theta) \sqrt{n}$ is at least as big as a binomial random variable $Q_{n}$ with parameters $N_{n}$ and $\sigma$. Since $N_{n}$ itself is $\operatorname{Geom}\left(p_{n}\right)$ distributed with parameter $p_{n}=\exp (-\theta(1-\theta) \sqrt{n}+\theta)$, straightforward calculation gives that the distribution of $Q_{n}$ is a mixture:

$$
Q_{n}=\left\{\begin{array}{ccc}
\operatorname{Geom}\left(\frac{p_{n}}{\sigma+(1-\sigma) p_{n}}\right) & \text { with weight } & \frac{\sigma}{\sigma+(1-\sigma) p_{n}} \\
0 & \text { with weight } & \frac{(1-\sigma) p_{n}}{\sigma+(1-\sigma) p_{n}}
\end{array}\right.
$$

Let us turn to the estimation of the probability that a fixed isolated vertex can considerably increase its degree in a time interval of length $\theta \sqrt{n}$.

How fast is the convergence to the stationary distribution? This can be answered easily by coupling. Let us start two degree processes, one from the stationary distribution, and another one from an isolated vertex (i.e., from degree $=0$ ). Let the deletion of the vertex in question be governed by the same Poisson process in both cases. After the first deletion stick the two processes together. Then the probability that the two processes differ after time $t$ is at most $e^{-(1-\theta) t}$. Hence the same bound is valid for the total variation distance of the degree distribution at time $t$ from the stationary one.

Consequently, if a vertex is isolated at time $(1-\theta) \sqrt{n}$, then the probability that its degree at time $\sqrt{n}$ is larger than $k_{n}$, is at least

$$
\pi_{n}=\sum_{k=k_{n}}^{\infty} q_{k}-\exp (-\theta(1-\theta) \sqrt{n}) .
$$

If $k_{n}$ is specified in such a way that

$$
\sum_{n=1}^{\infty} \frac{p_{n}}{\pi_{n}}<\infty
$$

holds, then $p_{n}=o\left(\pi_{n}\right)$, and we have

$$
\begin{aligned}
& P\left(M(\sqrt{n}) \leq k_{n}\right) \leq E\left(\left(1-\pi_{n}\right)^{Q_{n}}\right) \\
& \quad=\frac{(1-\sigma) p_{n}}{\sigma+(1-\sigma) p_{n}}+\frac{\sigma}{\sigma+(1-\sigma) p_{n}} \cdot \frac{\frac{p_{n}}{\sigma+(1-\sigma) p_{n}}\left(1-\pi_{n}\right)}{1-\frac{\sigma\left(1-p_{n}\right)}{\sigma+(1-\sigma) p_{n}}\left(1-\pi_{n}\right)} \sim \frac{p_{n}}{\sigma \pi_{n}} .
\end{aligned}
$$

The sum of these probabilities is convergent by supposition. Hence the BorelCantelli lemma implies that, almost surely, $M(\sqrt{n})>k_{n}$ if $n$ is large enough.

Finally, we have to show that $M(t)$ cannot decrease significantly between $\sqrt{n}$ and $\sqrt{n+1}$. Suppose $M(\sqrt{n})>k_{n}$. Choose a vertex with maximal degree and select $k_{n}$ from its neighbours. Let us compute the probability that more than $\varepsilon k_{n}$ of them will be deleted between $\sqrt{n}$ and $\sqrt{n+1}$. The number $Z$ of deleted vertices is binomial with parameters $k_{n}$ and

$$
1-\exp ((1-\theta)(\sqrt{n}-\sqrt{n+1})) \leq(1-\theta)(\sqrt{n}-\sqrt{n+1}) \leq 2(1-\theta) n^{-1 / 2} .
$$

By Hoeffding's inequality

$$
P\left(Z>\varepsilon k_{n}\right) \leq \exp \left(-2\left(\varepsilon-2(1-\theta) n^{-1 / 2}\right)^{2} k_{n}\right) .
$$


If, in addition to (17), sequence $\left(k_{n}\right)$ satisfies

$$
\sum_{n=1}^{\infty} \exp \left(-\varepsilon^{2} k_{n}\right)<\infty
$$

then the Borel-Cantelli lemma gives

$$
\min \{M(t): \sqrt{n} \leq t \leq \sqrt{n+1}\}>(1-\varepsilon) k_{n}
$$

if $n$ is sufficiently large. Consequently, with $n=\left\lfloor t^{2}\right\rfloor$ we have a.s. $M(t) \geq(1-\varepsilon) k_{n}$ for all sufficiently large $t$.

Let us specify $k_{n}$ in all three cases to meet conditions (17) and (18).

In the subcritical case let

$$
k_{n}=\frac{\theta(1-\theta)}{\log \gamma}(1-\varepsilon) \sqrt{n} .
$$

Then condition (18) is satisfied. Moreover, by (3) we have

$$
\pi_{n}=\exp \left(-(1+o(1)) \log \gamma \cdot k_{n}\right) \text {. }
$$

Hence

$$
\frac{p_{n}}{\pi_{n}}=\exp (-(1+o(1)) \theta(1-\theta) \varepsilon \sqrt{n}),
$$

thus condition (17) is satisfied as well. Consequently,

$$
M(t)>(1+o(1))(1-\varepsilon)^{2} \frac{\theta(1-\theta)}{\log \gamma} t=(1+o(1))(1-\varepsilon)^{2} \frac{1-\theta}{\log \gamma} \log N(t)
$$

if $t$ is sufficiently large.

In the critical case let

$$
k_{n}=\frac{(1-\varepsilon)^{2}}{64} n,
$$

then (18) is fulfilled. In addition, (4) implies

$$
\pi_{n}=\exp \left(-(1+o(1)) \frac{1-\varepsilon}{4} \sqrt{n}\right) .
$$

Therefore

$$
\frac{p_{n}}{\pi_{n}}=\exp \left(-(1+o(1)) \frac{\varepsilon}{4} \sqrt{n}\right),
$$

and requirement (17) is also met. Hence

$$
M(t)>(1+o(1)) \frac{(1-\varepsilon)^{3}}{64} t^{2}=(1+o(1)) \frac{(1-\varepsilon)^{3}}{16} \log ^{2} N(t)
$$

if $t$ is large enough.

Finally, in the supercritical case set

$$
k_{n}=\exp ((1-\varepsilon) \theta(2 \theta-1) \sqrt{n}) .
$$

Then (18) is satisfied. By (5) we can write

$$
\pi_{n}=k_{n}^{(1+o(1))(1-\beta)}=\exp (-(1+o(1))(1-\varepsilon) \theta(1-\theta) \sqrt{n}),
$$

from which it follows that

$$
\frac{p_{n}}{\pi_{n}}=\exp (-(1+o(1)) \varepsilon \theta(1-\theta) \sqrt{n}) .
$$

This produces a finite sum, thus (17) holds true. Consequently, with $n=\left\lfloor t^{2}\right\rfloor$,

$$
\begin{aligned}
M(t)>(1-\varepsilon) k_{n}=\exp ((1+o(1)) & (1-\varepsilon) \theta(2 \theta-1) t) \\
& =\exp ((1+o(1))(1-\varepsilon)(2 \theta-1) \log N(t)),
\end{aligned}
$$

if $t$ is sufficiently large. 
Due to deletions, in our graph there is no persistent hub in the sense of Krapivsky and Redner [20, 2001] or Galashin [14, 2014+] (namely, a single vertex which emerges forever as vertex of maximal degree), unlike in certain preferential attachment models [11, Dereich and Mörters, 2009], 21, Móri, 2005]. As a corollary to Theorems 3 and 4 , it follows that the index of the vertex with the maximal degree tends to infinity with time.

\section{REFERENCES}

[1] Backhausz, Á., Móri, T. F., Asymptotic properties of a random graph with duplication. To appear in J. Appl. Probab. 52(2) (2015)

[2] Backhausz, Á., Móri, T. F., Weights and degrees in a random graph model based on 3interactions. Acta Math. Hungar. 143(1) (2014), 23-43.

[3] Barabási, A-L., and Albert, R., Emergence of scaling in random networks, Science 286 (1999), 509-512.

[4] Bebek, G., Berenbrink, P., Cooper, C., Friedetzky, T., Nadeau, J. and Sahinalp, S. C., The degree distribution of the generalized duplication model. Theor. Comput. Sci., 369 (2006), 234-249.

[5] Bubeck, S., Mossel, E., Rácz, M. Z., On the influence of the seed graph in the preferential attachment model. Preprint. arXiv:1401.4849.

[6] N. Champagnat and A. Lambert, Splitting trees with neutral Poissonian mutations I: Small families, Stochastic Process. Appl. 122 (2012), no. 3, 1003-1033.

[7] N. Champagnat, A. Lambert and M. Richard, Birth and death processes with neutral mutations, Int. J. Stoch. Anal. 2012, Art. ID 569081, 20 pp.

[8] N. Champagnat and A. Lambert, Splitting trees with neutral Poissonian mutations II: Largest and oldest families, Stochastic Process. Appl. 123 (2013), no. 4, 1368-1414.

[9] Chung, F., Lu, L., Dewey, T. G., and Galas, D. J., Duplication models for biological networks, J. Comput. Biol., 16 (2003), 677-687.

[10] Cohen, N., Jordan, J., and Voliotis, M., Preferential duplication graphs, J. Appl. Probab., 47 (2010), 572-585.

[11] Dereich, S., Mörters, P., Random networks with sublinear preferential attachment: Degree evolutions, Elect. J. Probab., 14 (2009), Paper no. 43, 1222-1267.

[12] Dereich, S., Ortgiese, M., Robust analysis of preferential attachment models with fitness, Combin. Probab. Comput. 23(3) (2014), 386-411.

[13] Farczadi, L., Wormald, N., On the degree distribution of a growing network model. Preprint. arXiv:1401.0933.

[14] Galashin, P., Existence of a persistent hub in the convex preferential attachment model. Preprint. arXiv:1310.7513.

[15] Galambos, J., The Asymptotic Theory of Extreme Order Statistics, Wiley, New York, 1978.

[16] Hamdi, M., Krishnamurthy, V., Yin, G. G., Tracking the empirical distribution of a Markovmodulated duplication-deletion random graph. arXiv:1303.0050[cs.IT].

[17] Hermann, F., Pfaffelhuber, P., Large-scale behavior of the partial duplication random graph. Preprint. arXiv:1408.0904.

[18] Jordan, J., Randomised reproducing graphs. Electron. J. Probab., 16 (2011), 1549-1562.

[19] Kim, J., Krapivsky, P. L., Kahng, B. and Redner, S., Infinite-order percolation and giant fluctuations in a protein interaction network. Phys. Rev., E66: 055101(R), 2002.

[20] Krapivsky, L., Redner, S., Organization of growing random networks. Phys. Rev. E 63 (2001), no. 6,066123 .

[21] Móri, T. F., The maximum degree of the Barabási-Albert random tree. Combin. Probab. Comput., 14 (2005), 339-348.

[22] Móri, T. F., On a 2-parameter class of scale free random graphs. Acta Math. Hungar., 114 (2007), 37-48.

[23] Móri, T. F., Random multitrees. Studia Sci. Math. Hungar., 47 (2010), 59-80.

[24] Ostroumova, L., Ryabchenko, A., Samosvat, E., Generalized preferential attachment: tunable power-law degree distribution and clustering coefficient. In: Algorithms and Models for the Web Graph, Lecture Notes in Computer Science 8305 (2013), 185-202.

[25] Pastor-Satorras, R., Smith, E., and Solé, R. V., Evolving protein interaction networks through gene duplication. J. Theor. Biol., 222 (2003), 199-210.

[26] Karlin, S., Pinsky, M. A., An Introduction to Stochastic Modeling, fourth edition, Academic Press, 2010.

[27] Szegö, G., Orthogonal Polynomials, 4th edition, Amer. Math. Soc. Colloq. Publ., vol. 23, AMS, Providence, RI, 1975. 
[28] Thörnblad, E., Asymptotic degree distribution of a duplication-deletion random graph model. Preprint. arXiv:1408.4268v1.

[29] Yule, G. U., A mathematical theory of evolution, based on the conclusions of Dr. J. C. Willis, F.R.S., Philos. Trans. R. Soc. Lond. Ser. B., 213 (1925), 402-410.

[30] T. Vallier, Transition of the degree sequence in the random graph model of Cooper, Frieze, and Vera, Stoch. Models 29 (2013), no. 3, 341-352.

MTA Alfréd Rényi Institute of Mathematics, Budapest and Department of Probability Theory and Statistics, Eötvös Loránd University, Pázmány P. s. 1/C, H-1117 BudAPEST, Hungary

E-mail address: agnes@math.elte.hu

Department of Probability Theory and Statistics, Eötvös Loránd University, Pázmány P. S. 1/C, H-1117 Budapest, Hungary

E-mail address: mori@math.elte.hu 